\section{(6) OPEN ACCESS}

\title{
A novel somatic mutation achieves partial rescue in a child with Hutchinson-Gilford progeria syndrome
}

\author{
Daniel Z Bar, ${ }_{1}^{1}$ Martin F Arltt, ${ }^{2}$ Joan F Brazier, ${ }^{3}$ Wendy E Norris, ${ }^{4}$ Susan E Campbell, ${ }^{3}$ \\ Peter Chines, ${ }^{1}$ Delphine Larrieu, ${ }^{5}$ Stephen $\mathrm{P}$ Jackson, ${ }^{5}$ Francis $\mathrm{S}$ Collins, ${ }^{1}$ \\ Thomas W Glover, ${ }^{2}$ Leslie B Gordon ${ }^{4,6}$
}

\begin{abstract}
- Additional material is published online only. To view please visit the journal online (http://dx.doi.org/10.1136/ jmedgenet-2016-104295)
\end{abstract}

'National Human Genome Research Institute, National Institutes of Health, Bethesda, Maryland, USA

2Department of Human Genetics, University of Michigan, Ann Arbor, Michigan, USA ${ }^{3}$ Center for Gerontology and Health Care Research, Brown University, Providence, Rhode Island, USA

${ }^{4}$ Department of Pediatrics, Hasbro Children's Hospital, Providence, Rhode Island, USA ${ }^{5}$ Department of Biochemistry, The Gurdon Institute, University of Cambridge, Cambridge, UK ${ }^{6}$ Warren Alpert Medical School of Brown University, Providence, Rhode Island, USA

\section{Correspondence to}

Dr Leslie B Gordon, Department of Pediatrics, Hasbro Children's Hospital, 593 Eddy Street, Providence, RI 02903, USA; Leslie_Gordon@brown.edu

DZB, MFA and JFB contributed equally.

Received 06 September 2016 Revised 03 November 2016 Accepted 11 November 2016 Published Online First

5 December 2016

CrossMark

To cite: Bar DZ, Arlt MF,

Brazier JF, et al. J Med Genet 2017:54:212-216.

\begin{abstract}
Background Hutchinson-Gilford progeria syndrome (HGPS) is a fatal sporadic autosomal dominant premature ageing disease caused by single base mutations that optimise a cryptic splice site within exon 11 of the LMNA gene. The resultant disease-causing protein, progerin, acts as a dominant negative. Disease severity relies partly on progerin levels.
\end{abstract}

Methods and results We report a novel form of somatic mosaicism, where a child possessed two cell populations with different HGPS disease-producing mutations of the same nucleotide-one producing severe HGPS and one mild HGPS. The proband possessed an intermediate phenotype. The mosaicism was initially discovered when Sanger sequencing showed a c.1968+2T>A mutation in blood DNA and a c.1968 $+2 T>C$ in DNA from cultured fibroblasts. Deep sequencing of DNA from the proband's blood revealed $4.7 \%$ c. $1968+2 T>C$ mutation, and $41.3 \%$ c. 1968 $+2 \mathrm{~T}>\mathrm{A}$ mutation.

Conclusions We hypothesise that the germline mutation was C.1968+2T>A, but a rescue event occurred during early development, where the somatic mutation from $A$ to $C$ at $1968+2$ provided a selective advantage. This type of mosaicism where a partial phenotypic rescue event results from a second but milder disease-causing mutation in the same nucleotide has not been previously characterised for any disease.

\section{INTRODUCTION}

Hutchinson-Gilford progeria syndrome (HGPS or Progeria) is an ultra-rare fatal syndrome of segmental premature ageing, with death due primarily to heart attacks at an average age of 14.7 years. ${ }^{1}$ Progressive failure to thrive, sclerodermatous skin, lipodystrophy, skeletal dysplasia, joint contractures and premature atherosclerosis with resultant strokes and heart attacks ensue postnatally. Diagnosis is based on clinical features plus detection of specific autosomal dominant mutations in LMNA. ${ }^{2}$ Approximately $90 \%$ of the time, HGPS is caused by a de novo heterozygous $\mathrm{C}>\mathrm{T}$ mutation at nucleotide 1824 within exon 11 (classic HGPS). This activates a cryptic splice donor site at nucleotides 1819-1825, resulting in an mRNA that deletes $150 \mathrm{nt}$ (figure 1A) and codes for a protein (called 'progerin') that is missing 50 amino acid residues near the $\mathrm{C}$-terminus. In a minority of patients, a progerin-producing mutation occurs in the splice donor at the beginning of intron 11 (non-classic HGPS); this also activates the cryptic splice donor in the exon 11 at nucleotides 18191825 (figure 1A). Progerin, the common protein product in all of these mutations, is a permanently farnesylated, aberrant lamin A protein that acts as a dominant negative, accelerating senescence of cells that express it. ${ }^{3}$ The severity and rate of disease progression in HGPS is reflected at least in part by the abundance of progerin, ${ }^{4}$ which in turn reflects the consequence of the specific germline mutation on spliceosome recognition of the cryptic donor. Progerin mRNA is transcribed from the classic HGPS allele around $80 \%$ of the time, from intron 11 splice donor mutations at varying rates depending on how severely the mutation interferes with the $5^{\prime}$ splice site consensus G/GTRAGT sequence, ${ }^{5}$ and from a normal LMNA gene less than $2 \%$ of the time,${ }^{6}$ but this isoform is produced in greater abundance as normal cells approach senescence. ${ }^{6}$ Progerin expression drives nuclear morphological abnormalities, mitochondrial dysfunction, defects in DNA repair, premature senescence ${ }^{7}$ and a variety of epigenetic alterations including global chromatin changes and misregulated gene expression. ${ }^{8}$ There are two documented sibling occurrences, both presumably stemming from parental mosaicism, where one phenotypically normal parent has germline mosaicism for cells with the classic HGPS mutation (c. 1824 G>T; p. G608G). ${ }^{9}{ }^{10}$ Here we report the first diagnosed occurrence of mosaicism in a child with HGPS. This type of mosaicism is exceptional in that rather than simple mosaicism for normal and mutant alleles, the proband is mosaic for cells with two distinct disease-causing mutations of the same nucleotide. This type of occurrence has not been previously reported for any disease.

\section{MATERIALS AND METHODS \\ Patients}

This study was approved by the Institutional Review Boards of Rhode Island Hospital and Brown University, Providence, RI, as part of the Progeria Research Foundation (PRF) Cell and Tissue Bank and PRF Medical and Research Database programmes. All patients were diagnosed with HGPS based on phenotypic expression of the 
A

Normal

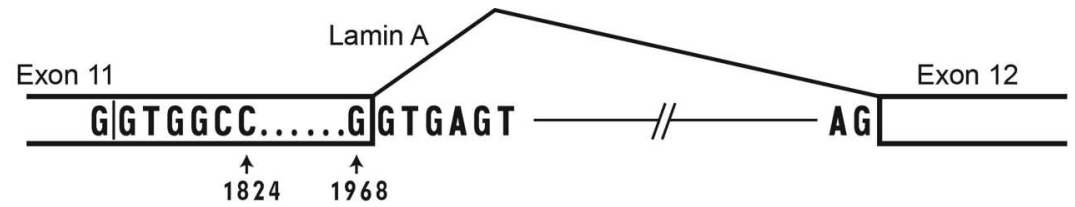

[Classic HGPS]
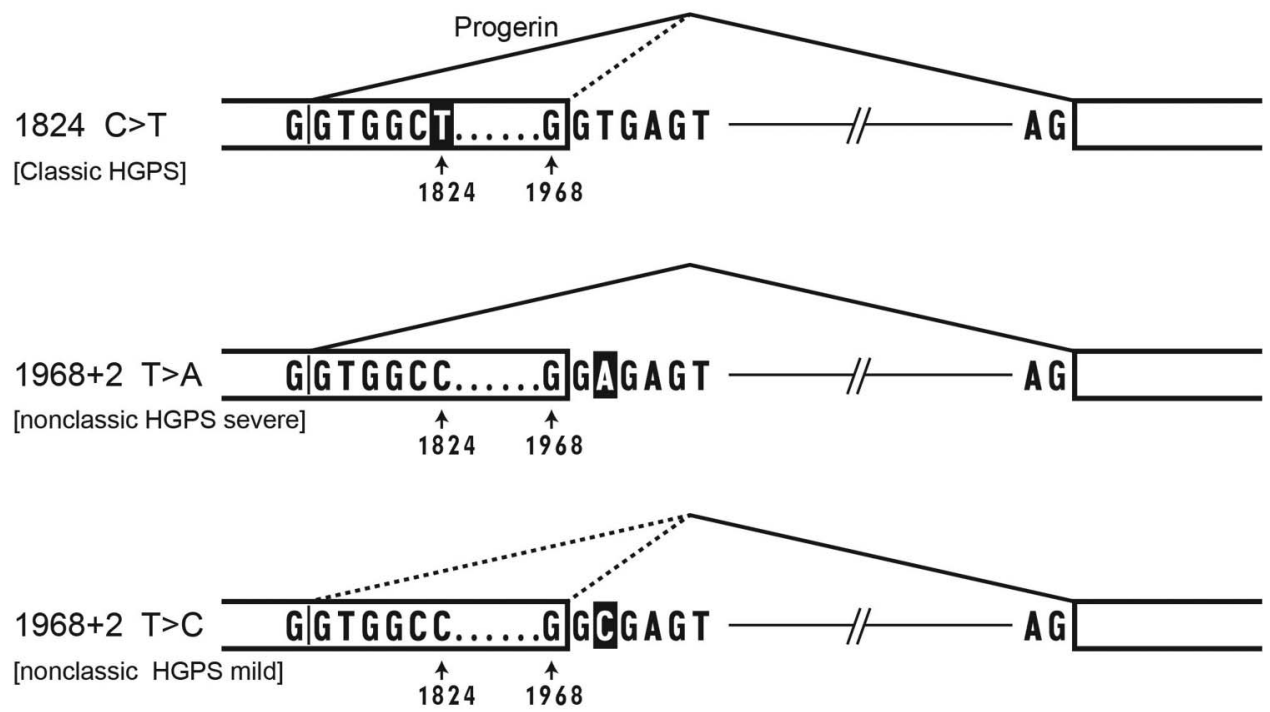

B

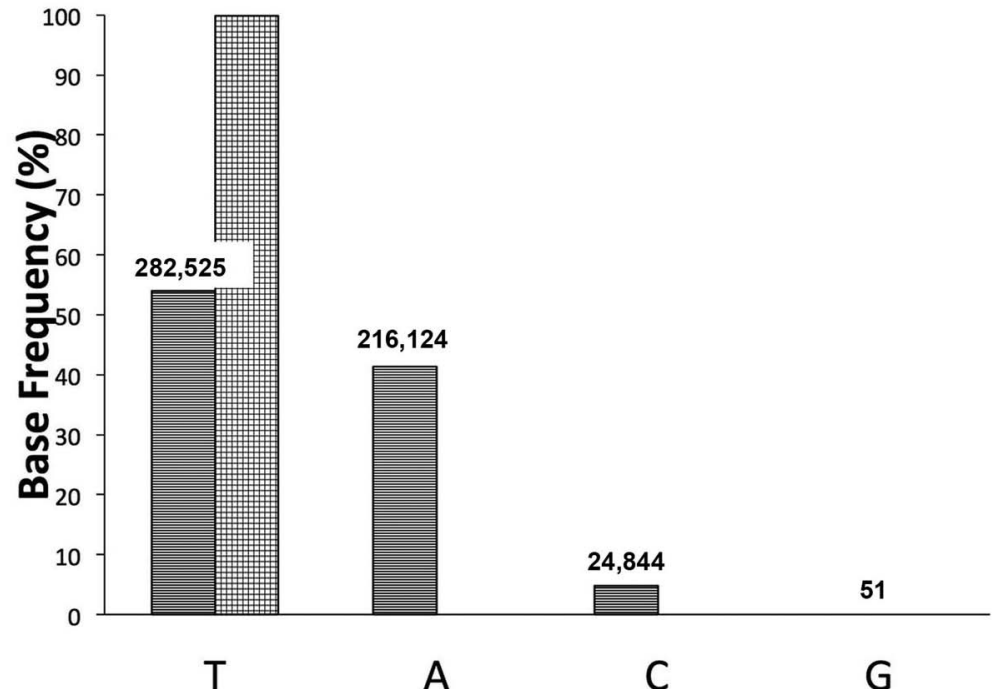

Figure 1 (A) Normal sequence and three Hutchinson-Gilford progeria syndrome (HGPS) mutations in the LMNA gene that affect exon 11 and intron 11. In the normal version, splicing occurs primarily from the normal splice donor at the beginning of intron 11, which is an excellent match to the consensus sequence G/GTRAGT, where the GT at the beginning of the intron is most critical, and R=A or G. The classic HGPS mutation activates a cryptic splice site just upstream of position 1824 in exon 11; the C>T HGPS mutation makes that splice donor a better match to the consensus at the +6 position. The bottom two lines show the consequences of mutations in the +2 position of intron 11, the topic of this report. When the 1968 +2 position is $A$ instead of $T$, the normal splice donor is predicted to be completely inactivated. When that position is $C$, some residual function of the normal splice is likely, as GC can substitute for GT in certain normal splice donors. ${ }^{16}$ All three of the mutant versions are predicted to lead to production of varying amounts of an mRNA that lacks $150 \mathrm{nt}$ of coding region, and thus codes for progerin, a toxic protein that is missing 50 amino acid residues. Mutations in the intron 11 splice donor have also been reported to produce an additional mRNA that completely deletes exon 11, but the protein product of that event has not thus far been detectable. ${ }^{5}$ (B) LMNA deep sequencing demonstrates genomic DNA base frequency at position1968+2, in white blood cells from patient DB386 (stripes), and cultured fibroblasts from father of DB386 1968+2 (checkers). Numbers of reads above each bar.

disease and confirmed LMNA mutational analysis performed by The Progeria Research Foundation Diagnostics Programme (http://www.progeriaresearch.org) or confirmed genetic analysis from medical records. Informed consent was obtained from all participants' parents, using translators when appropriate.
PRF cell and tissue bank samples

Blood-derived DNA and all fibroblast lines were obtained from the PRF Cell and Tissue Bank (http://www.progeriaresearch. org). Fibroblasts were cultured in Dulbecco's Modified Eagle Medium (DMEM) containing 15\% fetal bovine serum and 1X 
GlutaMAX (Gibco, Grand Island, New York, USA), 100 U/mL of penicillin and streptomycin, in $5 \% \mathrm{CO}_{2}$ at $37^{\circ} \mathrm{C}$. Fibroblast line identities were as follows, with patient IDs in parentheses: PSADFN386 (DB386), PSADFN392 (DB392), PSADFN423 (DB423), PSMDFN387 and PSFDFN388 (parents of DB386); PSMDFN393 and PSFDFN394 (parents of DB392). Population doubling time (DT) was calculated as follows: $\mathrm{DT}=\mathrm{T} \ln 2 / \ln (\mathrm{Xe} /$ $\mathrm{Xb}$ ), where $\mathrm{T}$ is the incubation time, $\mathrm{Xb}$ is the cell number at the initiation of the incubation time and $\mathrm{Xe}$ is the cell number at end of the incubation time.

\section{Sanger sequencing}

Using patient blood and cultured fibroblast genomic DNA (gDNA), the full coding region of the LMNA exon 11 and the flanking $\sim 20$ bases of non-coding sequence were sequenced, and compared with the reference sequence. gDNA was extracted from white blood cells (WBCs) or fibroblasts, using the DNeasy Blood \& Tissue Kit (Qiagen). PCR conditions and primers used for LMNA coding sequence amplification were for exon 11: forward gcacagaaccacaccttcct, reverse ggtgggctgtctaggactca. PCR amplifications were performed with $50 \mathrm{ng}$ of gDNA using specific primers with the following programme: a denaturation step at $94^{\circ} \mathrm{C}$ for $3 \mathrm{~min}$, followed by 35 cycles composed of denaturation at $94^{\circ} \mathrm{C}$ for $45 \mathrm{~s}$, hybridisation at $63^{\circ} \mathrm{C}$ for $45 \mathrm{~s}$, elongation at $72^{\circ} \mathrm{C}$ for $1 \mathrm{~min}$ and a final elongation step of $3 \mathrm{~min}$.

\section{Proband and paternity validation}

The identity and origin of DNA samples derived from proband blood and cultured fibroblasts and from cultured parental fibroblasts was performed using the Sequenom MassARRAY iPLEX Platform (Agena Bioscience) for analysis of 36 SNP loci and AmpFLSTR Identifiler Plus PCR Amplification Kit (ThermoFisher Scientific) for amplification of 16 short tandem repeat (STR) loci according to the manufacturer's instructions.

\section{Deep sequencing}

gDNA was extracted using the Puregene Blood Core Kit A (Qiagen). The relevant genomic section was amplified for four cycles of PCR with primers containing a genomic binding site, unique molecular identifiers and Illumina primer recognition site. Controls running for a higher cycle number showed a single band at the expected size. PCR product was run on a $2 \%$ agarose gel, and DNA at the expected size was extracted and amplified further by PCR with primers binding to the Illumina primer recognition site. All PCR reactions were performed using Phusion high-fidelity DNA polymerase (New England Biolabs (NEB)). Sequencing was performed by $250 \mathrm{bp}$ paired-end sequencing on a MiSeq instrument (Illumina). Only reads showing an exact match $10 \mathrm{bp}$ before and after the analysed base were kept. Reads showing only a single strand, low quality or a mismatch either within a pair or within a unique molecular identifier (UMI) cluster were excluded from the analysis. After filtration, over $0.5 \mathrm{M}$ reads per analysed site remained.

\section{RESULTS}

The female proband, DB386, was diagnosed with HGPS at age 10 months (figure 2), with typical early-stage features such as prominent scalp veins, lipodystrophy, short stature and typical skin signs. ${ }^{5}$ Compared with classic HGPS, she experienced hair loss but not the total alopecia pathognomonic of classic HGPS, ${ }^{11}$ mandibular recession that is less pronounced than classic HGPS $^{12}$ and milder than expected joint contractures. Proband birth weight and length patterns were similar to classic HGPS. ${ }^{13}$ Her birth weight was just below the third centile, and stayed below the third centile thereafter; her stature for age was initially normal, and fell below the third centile at age 12 months and thereafter. However, the proband displayed better growth than expected for classic HGPS as she aged. Whereas children with classic HGPS display an average rate of weight and height gain of $0.44 \mathrm{~kg} /$ year $^{13}$ and $3.58 \mathrm{~cm} /$ year, ${ }^{14}$ respectively, after age 2 years, the proband's growth rates were $0.56 \mathrm{~kg} /$ year and $5.8 \mathrm{~cm} /$ year after age 2 years. Whereas average weight and height for classic HGPS at age 5.9 years are $11.3 \mathrm{~kg}^{13}$ and $90 \mathrm{~cm},{ }^{14}$ respectively, the proband measured $11.7 \mathrm{~kg}$ and $97.2 \mathrm{~cm}$ at this age.

Regarding cardiovascular disease, in classic HGPS, carotid artery plaque can be noted at any age, blood pressure is elevated in about half of patients when adjusting for height age and hyperinsulinemia or insulin resistance is present in 52\% and $36 \%$ of patients, respectively. ${ }^{15}$ ECGs are often normal until late in life. The proband was first noted to have a left-sided carotid artery plaque at age 4.2 years. But at age 5.9 years, she demonstrated normal blood pressure, no insulinemia or insulin resistance, and no ECG abnormalities. Thus, while this patient was originally described as having a severe phenotype, ${ }^{5}$ our longitudinal clinical evaluations clearly demonstrate a phenotype that is milder than classic HGPS.

Standard Sanger sequencing of LMNA exon 11 DNA from blood-derived WBCs and cultured skin fibroblasts sequenced at passages 1, 3 and 8 detected differing progerin-producing mutations in the same nucleotide of the exon 11 intronic splice donor site (see online supplementary figure). The blood-derived DNA displayed c. $1968+2 \mathrm{~T}>\mathrm{A}$, while all three fibroblastderived DNA isolates displayed c. $1968+2 \mathrm{~T}>\mathrm{C}$, both heterozygous progerin-producing mutations.

We validated the proband origin of the blood and fibroblast DNA as well as paternity and maternity, using Sequenom 36-SNP MassARRAY Genotyping and Identifier-plus (Combined DNA Index System (CODIS)) microsatellite repeat PCR amplification (see online supplementary tables). Sequencing of parental LMNA exon 11 blood-derived WBC DNA revealed no mutations (see online supplementary figure).

To test for mosaicism, deep sequencing of the exon/intron 11 boundary in blood DNA was subsequently performed (figure 1B). The DNA sequence reads revealed roughly 50\% normal LMNA sequence, but $4.7 \%$ c. $1968+2 \mathrm{~T}>\mathrm{C}$ mutation and $41.3 \%$ c. 1968 $+2 \mathrm{~T}>\mathrm{A}$ mutation. Paternal cultured fibroblast DNA contained only the normal sequence, $1968+2 \mathrm{~T}$. An internal control proband comparator site at position $1968+6 \mathrm{~T}$ also contained only the normal sequence (537902 reads, 99.8\% of total).

To understand the relationship of the patient's phenotype to this apparent mosaicism, we assessed two additional patients, each with one of the mutations found in DB386 (see online supplementary figure). The female patient DB423 was heterozygous for the c. $1968+2 \mathrm{~T}>\mathrm{A}$ mutation and had a severe disease phenotype (figure 2). Progeria was suspected at birth clinically, and diagnosed at age 9 months by genetic testing. Sclerodermatous skin, nail dystrophy, lipodystrophy and failure to thrive were present at birth. Hair loss began at age 3 months. Mandibular recession and joint contractures were severe. She died of atherosclerotic cardiovascular disease with aggravating pneumonia at age 3.5 years. The male patient DB392 was heterozygous for the c. $1968+2 \mathrm{~T}>\mathrm{C}$ mutation and had a considerably milder progeria disease phenotype. His initial misdiagnosis at age 2.9 years was ectodermal dysplasia due to thick skin and brittle nails. Progeria was first suspected at age 7.0 years clinically, and diagnosed at age 7.1 years by genetic testing. His clinical history includes decreased weight initially appearing at age 
A

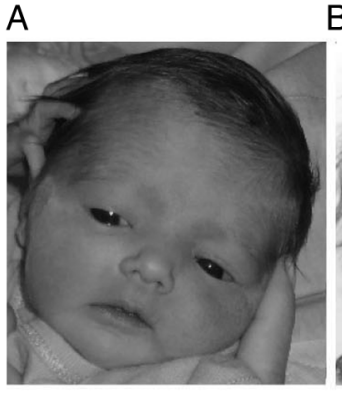

E

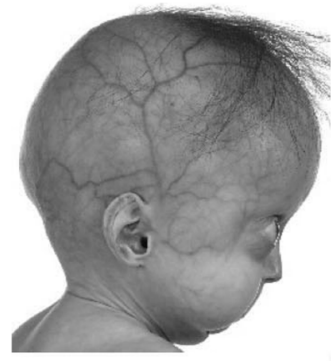

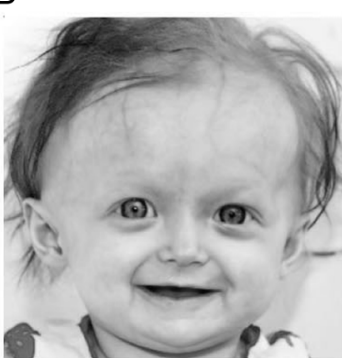

C

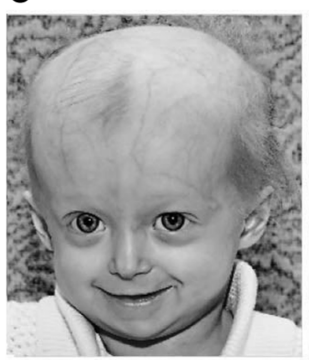

D

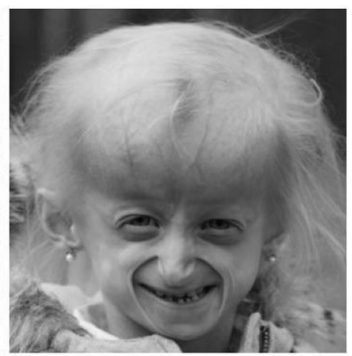

$\mathrm{F}$

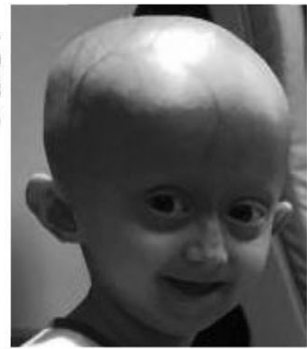

G

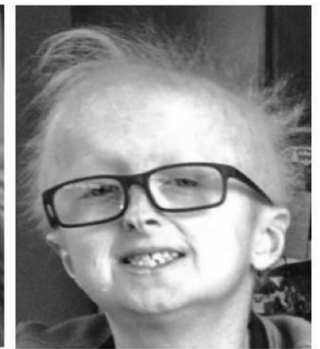

Figure 2 Proband DB386 at ages (A) 1 day (B) 1 year (C) 19 months (D) and 5.4 years demonstrating progressive mandibular recession, severe but subtotal alopecia, and lipodystrophy. (E) DB423 at age 1.2 years demonstrating severe mandibular recession, early alopecia and severe lipodystrophy (F) female with classic Hutchinson-Gilford progeria syndrome at age 5.4 years demonstrating total alopecia severe lipodystrophy and moderate mandibular recession. (G) DB392 at age 11.5 years demonstrating no mandibular recession, moderate alopecia and moderate lipodystrophy.

1.3 years with an overall weight at the third centile, nail dystrophy, skin signs and sparse thin hair without total alopecia initially developing at age 2.9 years. Stature for age was initially normal, and fell below the third centile at age 4 years and thereafter. He displayed no mandibular recession, normal dentition and normal cardiovascular function as of age 11 years.

Patient and parental fibroblast culture population doubling times were assessed through passage 8 . Doubling times were between 2 and 6 days for all cultures until passage 6 . The more severe patient's fibroblasts (DB423) then increased to 11.1 and 28.8 days with successive passages, whereas cultures from the milder phenotype patient (DB392) and the patient with mosaicism (DB386) maintained doubling times below 6 days throughout. Parental fibroblast culture population doubling times were between 2 and 6 days for all cultures throughout.

\section{DISCUSSION}

Based on these phenotype/genotype comparisons, we hypothesise that a partial rescue event occurred during fetal development in patient DB386, where the germline c. $1968+2 \mathrm{~T}>\mathrm{A}$ mutation was secondarily mutated to c. $1968+2 \mathrm{~T}>\mathrm{C}$. Approximately $0.7 \%$ of normal splice donors have GC instead of GT. ${ }^{16}$ Although such GC donors have a mismatch with the U1 snRNA that catalyses the splice event, they still can function when the rest of the splice consensus is a close match to the G/ GTRAGT consensus, as is true in this situation. Thus, the presence of $\mathrm{C}$ in the +2 position has provided some residual function to the intron 11 splice donor (figure $1 \mathrm{~A}$ ), so that the cryptic donor in exon 11 is less activated. Hence, while progerin is still produced, the disease phenotype in the proband DB386 is significantly milder than the patient with the c. $1968+2 \mathrm{~T}>\mathrm{A}$ mutation, slightly milder than the classic HGPS phenotype ${ }^{14}$ and significantly more severe than the patient with the c.1968 $+2 \mathrm{~T}>\mathrm{C}$ mutation.

Fibroblasts culture doubling times support a growth advantage for the milder phenotype mutation. Cultured fibroblasts from the severely affected c. $1968+2 \mathrm{~T}>\mathrm{A}$ patient were extremely difficult to grow, reaching a population DT of 28.8 days by passage 8 , while those of the patients with the milder c. $1968+2 \mathrm{~T}>\mathrm{C}$ mutation and the patient with mosaicism grew significantly better. Thus, it is likely that in vivo or in vitro clonal selection during fibroblast growth from the patient with mosaicism resulted in a selective growth advantage for the cells containing the milder mutation, causing Sanger sequencing of the fibroblasts from the patient with mosaicism to detect only the milder mutation in these cells. This elimination of the more severe c. $1968+2 \mathrm{~T}>\mathrm{A}$ mutation did not occur in WBCs, presumably because they express very low levels of lamin A and are therefore not subject to selection.

The possibility that the mutations occurred in the opposite order could also be considered, but fits the data much less well. Theoretically, a somatic mutation from c. $1968+2 \mathrm{~T}>\mathrm{C}$ to c. $1968+2 \mathrm{~T}>\mathrm{A}$ in a haematopoietic progenitor cell, where the absence of LMNA expression would prevent negative selection, might be possible-but in that instance, one would expect the phenotype of DB386 to match the milder phenotype of DB392.

Close inspection of the data from DB386 WBCs in figure 1B shows that the summed proportion of $\mathrm{C}$ and $\mathrm{A}$ reads $(46 \%)$ is slightly less than the $\mathrm{T}$ reads (54\%), whereas one would have predicted this should be $50-50$. The most likely explanation is a subtle bias in PCR amplification efficiency between the three alleles being tested. We cannot exclude, however, that a second somatic event might have occurred in the precursor of WBCs: either a back mutation to the $\mathrm{T}$ allele or a complete deletion of the mutant LMNA locus.

The serendipitous discovery of mosaicism in patient DB386 suggests that similar occurrences may go undetected, as there is no routine testing for identification of such rare events. As an embryo develops from a single cell to a human being, a large number of cell divisions occur, providing both the means and the selective pressure for adaptive mutations. In addition, it is possible that this is a particularly unstable nucleotide position in 
somatic tissue when mutated. Future larger systematic studies should be undertaken to identify mosaicism frequency in HGPS and other single gene disorders.

This type of mosaicism, where a rescue event causes a second but milder disease-causing mutation instead of a reversion mutation to the normal genotype, has not been previously characterised for any disease. We predict that next-generation sequencing, targeted deep sequencing or digital droplet PCR of patients with genetic disorders may uncover other examples of this form of genetic adaptation. In some cases, such as gene therapy or RNA therapeutics, therapy may be altered by awareness of an event such as this. In addition, therapeutic efficacy might differ for patients with these types of mosaicisms.

Acknowledgements The authors are extremely grateful to the children and families for participation in this study. They thank the University of Michigan Sequencing Core for assistance. They also thank Drs. Thorsten Marquardt and Janine Reunert for additional assistance.

Contributors Each author listed on the manuscript has participated in editing the manuscript, has seen and approved the submission of this version of the manuscript and takes full responsibility for the manuscript. In addition, DZB and MFA conducted, analysed and interpreted the deep sequencing experiments; JFB and SEC acquired and analysed phenotypic data; WEN acquired and processed all patient samples, and analysed data. PC and TWG performed and interpreted Sanger sequencing and paternity validation studies; SPJ and DL commissioned the sequencing studies that led to the initial identification of genetic mosaicism in the patient. FSC and TWG contributed to study design and data analysis. LBG was responsible for primary study oversight and design, data acquisition, data analysis and interpretation, and primary writing of the manuscript.

Funding Progeria experiments were funded by The Progeria Research Foundation grants PRF-2002-CB and PRF-2002-MRD (JFB, WEN, SEC, LBG), and by the Medical Research Council UK grant MR/L019116/1 (DL). Core and general laboratory grants are as follows: Kilguss Research Core of Women \& Infants Hospital of Rhode Island through an Institutional Development Award from the NIGMS of the $\mathrm{NIH}$ (P30GM114750), intramural funds to the NHGRI (ZIA-HG200305), Cancer Research UK programme grant C6/A18796 and Wellcome Trust (WT092096).

Competing interests None.

Patient consent Obtained.

Ethics approval Rhode Island Hospital.

Provenance and peer review Not commissioned; externally peer reviewed.

Open Access This is an Open Access article distributed in accordance with the terms of the Creative Commons Attribution (CC BY 4.0) license, which permits others to distribute, remix, adapt and build upon this work, for commercial use, provided the original work is properly cited. See: http://creativecommons.org/licenses/ by/4.0/

\section{REFERENCES}

1 Gordon LB, Massaro J, D'Agostino RB Sr, Campbell SE, Brazier J, Brown WT, Kleinman ME, Kieran MW, Progeria Clinical Trials Collaborative. Impact of farnesylation inhibitors on survival in hutchinson-gilford progeria syndrome. Circulation 2014;130:27-34.

2 Gordon LB, Brown WT, Collins FS. Hutchinson-gilford progeria syndrome. In: Pagon RA, Adam MP, Ardinger HH, Bird TD, Dolan CR, Fong CT, Smith RJH, Stephens K, eds. Seattle, WA: Genereviews(r), 2015. https://www.ncbi.nlm.nih.gov/books/ NBK1121/ (accessed online Nov 2016).

3 Eisch V, Lu X, Gabriel D, Djabali K. Progerin impairs chromosome maintenance by depleting cenp-f from metaphase kinetochores in hutchinson-gilford progeria fibroblasts. Oncotarget 2016:7:24700-18.

4 Hisama FM, Lessel D, Leistritz D, Friedrich K, McBride KL, Pastore MT, Gottesman GS, Saha B, Martin GM, Kubisch C, Oshima J. Coronary artery disease in a werner syndrome-like form of progeria characterized by low levels of progerin, a splice variant of lamin a. Am J Med Genet A 2011;155A:3002-6.

5 Harhouri K, Navarro C, Baquerre C, Da Silva N, Bartoli C, Casey F, Mawuse G, Doubaj Y, Lévy N, De Sandre-Giovannoli A. Antisense-based progerin downregulation in hgps-like patients' cells. Cells 2016;5:31.

6 Cao K, Blair CD, Faddah DA, Kieckhaefer JE, Olive M, Erdos MR, Nabel EG, Collins FS. Progerin and telomere dysfunction collaborate to trigger cellular senescence in normal human fibroblasts. J Clin Invest 2011;121:2833-44.

7 Gonzalo S, Kreienkamp R, Askjaer P. Hutchinson-gilford progeria syndrome: a premature aging disease caused by Imna gene mutations. Ageing Res Rev Published Online First 29 Jun 2016. doi: 10.1016/j.arr.2016.06.007

8 Arancio W, Pizzolanti G, Genovese SI, Pitrone M, Giordano C. Epigenetic involvement in hutchinson-gilford progeria syndrome: a mini-review. Gerontology 2014:60:197-203.

9 The progeria research diagnostic testing program. http://progeriaresearch.org/ diagnostic_testing.html (accessed 1 Aug 2016).

10 Wuyts W, Biervliet M, Reyniers E, D'Apice MR, Novelli G, Storm K. Somatic and gonadal mosaicism in hutchinson-gilford progeria. Am J Med Genet A 2005;135:66-8.

11 Rork JF, Huang JT, Gordon LB, Kleinman M, Kieran MW, Liang MG. Initial cutaneous manifestations of hutchinson-gilford progeria syndrome. Pediatr Dermatol 2014;31:196-202.

12 Ullrich NJ, Silvera VM, Campbell SE, Gordon LB. Craniofacial abnormalities in hutchinson-gilford progeria syndrome. AJNR Am J Neuroradiol 2012;33:1512-18.

13 Gordon LB, McCarten KM, Giobbie-Hurder A, Machan JT, Campbell SE, Berns SD, Kieran MW. Disease progression in hutchinson-gilford progeria syndrome: Impact on growth and development. Pediatrics 2007;120:824-33.

14 Merideth MA, Gordon LB, Clauss S, Sachdev V, Smith A, Perry M, Brewer C, Zalewski C, Kim H, Solomon B, Brooks B, Gerber L, Turner M, Domingo DL, Hart TC, Graf J, Reynolds J, Gropman A, Yanovski J, Gerhard-Herman M, Collins FS, Nabel EG, Cannon R, Gahl WA, Introne WJ. Phenotype and course of hutchinson-gilford progeria syndrome. N Engl J Med 2008:358:592-604.

15 Gerhard-Herman M, Smoot LB, Wake N, Kieran MW, Kleinman ME, Miller DT, Schwartzman A, Giobbie-Hurder A, Neuberg D, Gordon LB. Mechanisms of premature vascular aging in children with hutchinson-gilford progeria syndrome. Hypertension 2012;59:92-7.

16 Burset M, Seledtsov IA, Solovyev VV. Analysis of canonical and non-canonical splice sites in mammalian genomes. Nucleic Acids Res 2000;28:4364-75. 